\title{
Comprender el trabajo de los docentes a través de su interacción con los recursos de su enseñanza - una historia de trayectorias
}

\section{Understanding the work of teachers through their interaction with their teaching resources -a history of trajectories}

\section{Luc Trouche ${ }^{1}$}

\begin{abstract}
Resumen: En este artículo se muestra, a partir de un testimonio personal, cómo se entrelazan las historias tecnológicas, conceptuales, profesionales y humanas e ilustrar esta interrelación desde la trayectoria de un recurso. En la primera sección se presenta dicho testimonio. En la segunda se expone el marco conceptual en el que se sitúa actualmente nuestra investigación. En la tercera se introduce el problema matemático que sustenta nuestra reflexión. La cuarta sección se dedica a la aplicación de este problema en un contexto de formación de docentes en Francia, en 2002. La quinta se destina al análisis del mismo problema en el contexto de un seminario de investigación en México en 2017. En la sexta sección se destacan las tensiones relativas a la formación docente, entre "darles herramientas para apoyar su trabajo en el aula" y "darles tiempo para que, juntos, puedan expresar su creatividad". Este artículo retoma, en especial, los términos de un seminario que se llevó a cabo en el Instituto Freudenthal de Utrecht, por invitación de Paul Drijvers (Trouche 2018A).
\end{abstract}

Palabras clave: enfoque documental en la didáctica, creatividad matemática, formación de docentes, instrumentación, instrumentalización, sistema de recursos.

\begin{abstract}
This article shows, from a personal testimony, how the technological, conceptual, professional and human histories are intertwined and illustrate this interrelation from the trajectory of a resource. In the first section that testimony
\end{abstract}

Fecha de recepción: 30 de julio de 2018. Fecha de aceptación: 30 de agosto de 2018.

1 Instituto Francés de la Educación, Escuela Normal Superior de Lyon, luc.trouche@ens-lyon.fr orcid.org/ 0000-0001-5601-2930 
is presented. The second one exposes the conceptual framework in which our research is currently located. In the third, the mathematical problem that underlies our reflection is introduced. The fourth section is devoted to the implementation of this problem in a context of teacher training in France in 2002. The fifth section is intended to the analysis of the same problem in the context of a research seminar in Mexico in 2017. In the sixth section the tensions related to teacher training are highlighted, between "giving them tools to support their work in the classroom" and "giving them time so that, together, they can express their creativity". This article takes up, in particular, the terms of a seminar that was held at the Freudenthal Institute of Utrecht, at the invitation of Paul Drijvers (Trouche 2018A).

Keywords: Documentational approach to didactics, mathematical creativity, teacher training, instrumentation, instrumentalization, resource system

\section{LAS MÚLTIPLES Y ENTRELAZADAS RAZONES DE LAS GÉNESIS IDENTITARIAS}

Retomaré aquí la idea de Pastré (2005, p. 231) de un "polo en la organización de la actividad humana, que se estructura en torno a la experiencia adquirida y continuamente transformada por los actores". En ella se hace referencia (ibíd., p. 234) a la existencia de "génesis identitarias que expresan momentos particulares en la construcción de la experiencia". De estos momentos particulares existen tres en mi propia experiencia y me gustaría evocarlos aquí: el primero con la aparición de las calculadoras gráficas, el segundo con el surgimiento de las redes de calculadoras y el tercero con el nacimiento de las comunidades de profesores en línea (para mayor información acerca de esta génesis identitaria, véase Trouche, 2009).

\subsection{LA APARICIÓN DE LAS CALCULADORAS GRÁFICAS}

En mi memoria, el primer momento se caracterizó por la aparición de calculadoras gráficas en las aulas y la confrontación con fenómenos didácticos nuevos. Así pues, la figura 1 muestra una representación gráfica de la función 
$\ln (x)+10 \sin (x)$, que aparece principalmente como una función "oscilante" para los alumnos (Guin \& Trouche 1998). A la pregunta “¿Cuál es el límite de esta función en $+\infty$ ?", en un entorno de calculadoras gráficas, los alumnos responden en su mayoría "esta función no tiene límite", apoyando su respuesta en el carácter oscilante de la representación gráfica de la función. En ausencia de una calculadora gráfica, los estudiantes dependen principalmente de la descomposición de la función en la suma de dos funciones: $\ln (x)$ que tiende hacia $+\infty$, y de $\operatorname{seno}(x)$ que está limitada, y responden que la función $f$ tiene por limite a $+\infty$.
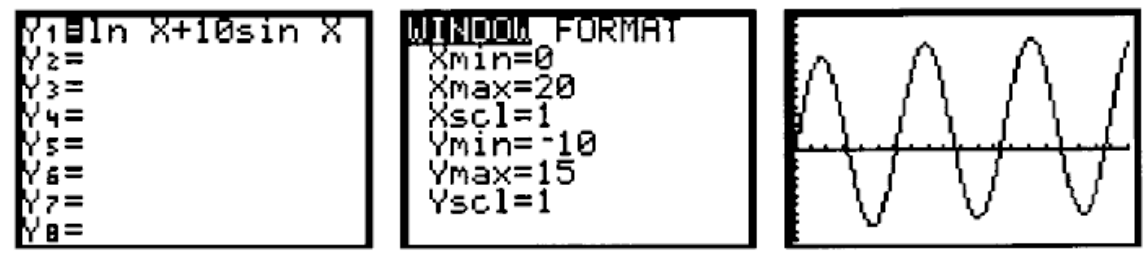

Figura 1. Capturas de pantalla de una calculadora para la representación de una función 'oscilante' (Guin \& Trouche 1998, p. 198).

Esta influencia que tienen algunos artefactos sobre el aprendizaje matemático me lleva, junto con otros investigadores, a repensar la relación entre instrumentación y conceptualización, que será la semilla del desarrollo del enfoque instrumental en la didáctica (Guin \& Trouche 1998), que pone de relieve la relación dialéctica entre los artefactos y sus usuarios: los artefactos condicionan la actividad estudiantil (éstos son los procesos de instrumentación), y los estudiantes en la práctica de su actividad determinada, adaptan los artefactos que adoptan (éstos son los procesos de instrumentalización). Esta relación dialéctica se desarrolla de manera diferente según los alumnos, lo que puede llevar a una gran dispersión de los procesos de conceptualización cuanto más grande sea la complejidad de los artefactos. En retrospectiva, me doy cuenta de que, en ese momento, me concebía principalmente como profesor (era maestro en una preparatoria), y consideraba a la enseñanza y al aprendizaje básicamente como hechos individuales. 


\subsection{EL SURGIMIENTO DE LAS REDES DE CALCULADORAS}

El segundo momento se caracterizó por la aparición de las redes de calculadoras gráficas, por ejemplo la tecnología Navigator, desarrollada por Texas Instruments (figura 2).

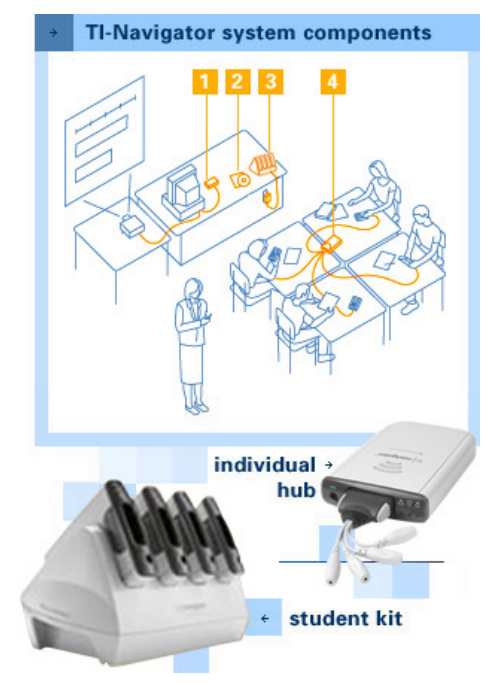

Figura 2. Tecnología TI-Navigator $($, que permite conectar cuatro calculadoras gráficas a un Hub conectado a la computadora del profesor.

Este tipo de tecnología le brinda al profesor los medios para tratar de reducir la dispersión del comportamiento estudiantil frente al tamaño reducido de la pantalla de las calculadoras: el conectar estas calculadoras a una red permite compartir los diferentes resultados propuestos por los estudiantes, posibilitando que esta diversidad sea una fuente de riqueza al servicio del aprendizaje. Idea que desarrollé durante el panel Connectivity and virtual networks for learning (Conectividad y redes virtuales para el aprendizaje) coordinado por Celia Hoyles en 2006, durante el estudio ICMI Mathematics education and digital technology: Rethinking the terrain (Educación matemática y tecnología digital: repensando el campo) (Hoyles, et al., 2010).

De hecho, esta tecnología ofrece nuevos medios para desarrollar la reflexión en torno a la orquestación instrumental de las situaciones matemáticas. Había introducido este concepto de orquestación instrumental en 2004, y luego lo reformulé en un trabajo conjunto con Paul Drijvers (Drijvers \& Trouche 2008). La 
orquestación instrumental se centraba en el manejo didáctico de los artefactos para la implementación de una situación matemática. La idea era pensar las configuraciones didácticas en clase (la manera en que se conectan los estudiantes y los artefactos) y, los mecanismos de análisis de estas configuraciones. Por lo tanto, una configuración didáctica emblemática es la del alumno sherpa (figura 3), la cual se basa en la visualización de la pantalla de la calculadora de uno de los alumnos en la pantalla del aula, desempeñando así el papel de mediador entre el maestro y los estudiantes.

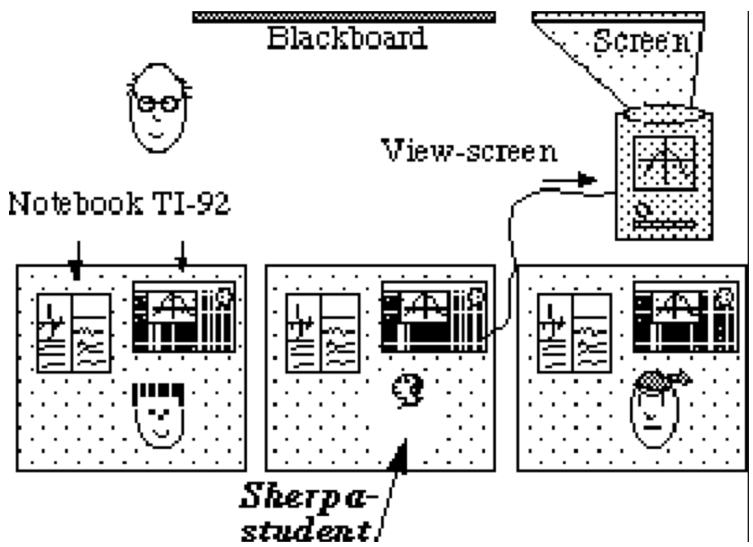

Figura 3. La configuración didáctica del alumno sherpa (Trouche 2004, p. 298).

Las redes de calculadoras y de otros dispositivos que conectan artefactos dentro del aula, proporcionan medios para repensar orquestaciones instrumentales, desde el punto de vista de la diversidad de las posibles configuraciones didácticas, como el concepto en sí mismo. En consecuencia, Drijvers, et al. (2010) proponen añadir, después de las configuraciones didácticas y sus modalidades de análisis, un nivel de rendimiento didáctico que corresponda a la implementación de las orquestaciones en el aula, y a los múltiples ajustes que el maestro y los estudiantes puedan realizar al calor del momento. En retrospectiva, me doy cuenta de que en ese momento me concebía principalmente como formador de docentes que consideraba todavía a la enseñanza como una responsabilidad individual, pero al aprendizaje como una responsabilidad primordialmente colectiva en donde el maestro desempeña un papel fundamental en el desarrollo del estudiantado. 


\subsection{El NACIMIENTO DE LAS COMUNIDADES DE DOCENTES EN LÍNEA}

El tercer momento se caracterizó por el desarrollo de Internet, y la creación de comunidades de docentes en línea. En Francia, este surgimiento es espectacular, con el desarrollo de una asociación de profesores de matemáticas, Sésamath (http://www.sesamath.net/), que agrupa sus recursos, diseña nuevos recursos colaborativamente y los sube a Internet, ofreciéndolos de forma gratuita a todos los profesores interesados (Gueudet \& Trouche 2009a, Rocha \& Trouche 2016). Se trata de una verdadera metamorfosis del trabajo docente que dispone de un número casi ilimitado de recursos y de medios eficaces para compartir su experiencia. Retrospectivamente me doy cuenta de que es en ese momento en que me concibo principalmente como investigador. El problema que me ocupa, en particular, ya no es apoyar el trabajo de los docentes sino comprender sus interacciones con los recursos de su enseñanza. Así pues, tanto la enseñanza como el aprendizaje aparecen esencialmente como hechos colectivos. A partir de esta evolución va a nacer una nueva línea de investigación, el enfoque documental en la didáctica, desarrollado en un trabajo conjunto con Ghislaine Gueudet (Gueudet \& Trouche 2009b), enfoque que es el tema de la siguiente sección.

Para concluir la primera sección, quisiera señalar que esta génesis identitaria de investigador no sólo es individual: es la marca individual de procesos más amplios, como habíamos estudiado en un texto de 2013 (Trouche, et al.), cuyo interés era, sobre la base de un estudio de la literatura de investigación de los años 2000 a 2008, en los Technology-Driven Developments and Policy Implications for Mathematics Education (Ios desarrollos impulsados por la tecnología y las implicaciones políticas para la educación matemática). Este estudio mostraba la amplia evolución de los temas de investigación a nivel internacional, la evolución de la tecnología hacia los recursos y, la evolución del trabajo individual de los docentes hacia las formas colectivas de su trabajo. Estos desarrollos no sólo están vinculados a la evolución tecnológica sino también a las dinámicas de las comunidades de investigación y a los cuestionamientos institucionales.

Finalmente, podemos observar que las herramientas de referencia de la Web también mantienen registrados los tres momentos que acabo de mencionar, en torno a los tres artículos fundamentales que cristalizaron las principales evoluciones de una perspectiva de investigador y, de las colaboraciones relacionadas, el enfoque instrumental en la didáctica en 1998, el concepto de orquestación instrumental en 2004 y el enfoque documental en la didáctica en 2009. 


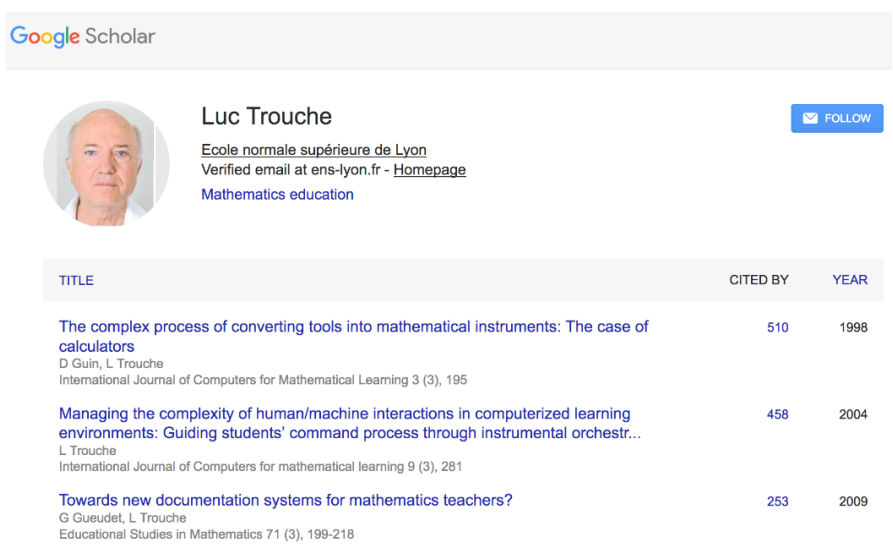

Figura 4. Las medidas, por Google Scholar, de la divulgación científica (declaración del 27 de julio de 2018).

\section{EL ENFOQUE DOCUMENTAL EN LA DIDÁCTICA}

Presento en esta sección el marco conceptual en el que ubico actualmente el enfoque documental en la didáctica dentro de mi investigación. Introducido a partir de una reflexión conjunta con Ghislaine Gueudet (Gueudet \& Trouche 2008), y desarrollado más adelante en un marco internacional (Gueudet, Pepin \& Trouche 2012), este enfoque también fue explorado en un contexto brasileño (Assis, Gitirana, \& Trouche 2018), así como en un contexto chino (Wang, Trouche, \& Pepin, 2018), e incluso mexicano (Sánchez 2010; Salinas-Hernández, Sacristán, \& Trouche, 2018). A continuación presento tres aspectos críticos de este enfoque: el concepto de recursos, la dialéctica de los recursos/documentos y el concepto de sistema de recursos.

\subsection{EL CONCEPTO CRÍTICO DE 'RECURSO'}

El concepto de 'recurso' es lo primero. Lo tomamos de las investigaciones de Adler (2012), dentro del campo de la educación matemática: un recurso es todo lo que sea susceptible de re-generar el trabajo de los docentes. Un maestro interactúa con los conjuntos de recursos; éstos son trabajados (adaptados, revisados, reorganizados...), a lo largo de procesos que articulan estrechamente el 
diseño y la implementación: es al conjunto de este trabajo lo que llamamos trabajo documental. La documentación se refiere tanto a este trabajo como a lo que produce. El trabajo documental es central en la actividad profesional de los profesores. Se encuentra en todos los aspectos de esta actividad, en todos sus espacios, en todos sus momentos. Observamos la actividad de los profesores en su conjunto y consideramos las interacciones con los recursos como elementos principales de esta actividad profesional; y es así como se impone esta amplia definición de recursos. No obstante, tomamos en cuenta la diversidad de ciertas características de los recursos: algunas son materiales, lo que permite un seguimiento más directo de las interacciones (apuntes tomados de un libro, cambios en un archivo); otras, intangibles, que son de acceso más difícil, pero sin embargo pueden desempeñar un papel decisivo en clase con los estudiantes, como las interacciones verbales o no verbales. Nos comprometemos a destacar, en nuestros análisis, las especificidades pertinentes, en particular las de los recursos materiales digitales. Desde que se introdujo este enfoque, infinidad de investigaciones han intentado esclarecer este concepto de recurso, por ejemplo Hammoud (2012), distinguiendo entre los recursos madre (los que el docente recaba para preparar su curso) y los recursos hijo (los que el docente produce e implementa en su clase). Un proyecto nacional de investigación en Francia (ReVEA, https://www.ANR-revea.fr/) exploró la diversidad de recursos utilizados y desarrollados por los docentes en un tiempo (2014-2018) de transición digital (Trouche, et al. 2018). El lugar que ocupan los recursos digitales (Pepin, et al. 2017) traduce, y de hecho implica, cambios importantes (en particular: la evolución de la naturaleza y del papel de los libros de texto, los nuevos equilibrios entre usos y concepciones de recursos).

\subsection{DiALÉCTICA RECURSOS /DOCUMENTOS Y GÉNESIS DOCUMENTALES}

A continuación presentamos los conceptos básicos del enfoque documental. El punto de partida de éste es el enfoque instrumental (ya introducido $\S 1,1$ ), tal como fue desarrollado por Rabardel (1995) en ergonomía cognitiva y luego integrado en la didáctica de las matemáticas (Guin \& Trouche, 1998, Trouche, 2005), Rabardel distingue un artefacto, disponible para un cierto usuario, de un instrumento que este usuario construye a partir de ese artefacto, en el curso de una acción determinada. Este proceso de desarrollo, las génesis instrumentales (figura 5), se basa, para cierto individuo, en la apropiación y transformación del artefacto, para resolver un problema dado, por medio de una variedad 
de contextos de uso. A través de esta variedad de contextos se constituyen esquemas de uso del artefacto: un esquema (Vergnaud 2009) es una organización invariante de actividad, que incluye principalmente reglas de acción, está estructurado por invariantes operativas que se forjan a lo largo de, y conducen esta actividad en diferentes contextos encontrados para la misma clase de situaciones. Por consiguiente, se puede proponer una definición sintética: instrumento = artefacto + esquema. Este enfoque también distingue dos tipos de procesos entrelazados en el núcleo de las génesis instrumentales, los procesos de instrumentación (constitución de los esquemas de uso de los artefactos a su alcance) y los procesos de instrumentalización (por los cuales el sujeto pone los artefactos a su alcance). Las génesis instrumentales no deben pensarse como mecanismos de producción, con un principio y un final claramente identificables; son procesos que se extienden en el tiempo, incluyendo momentos de estabilidad y rupturas. En el sentido del término que tenemos aquí, génesis puede significar tanto el nacimiento como la transformación, pero nunca designa una creación ex nihilo.

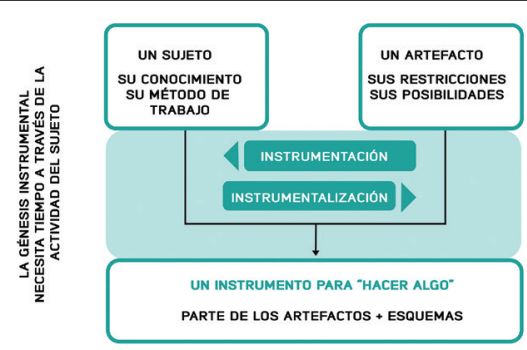

Figura 5. Modelización de una génesis instrumental (Trouche 2005, p. 144).

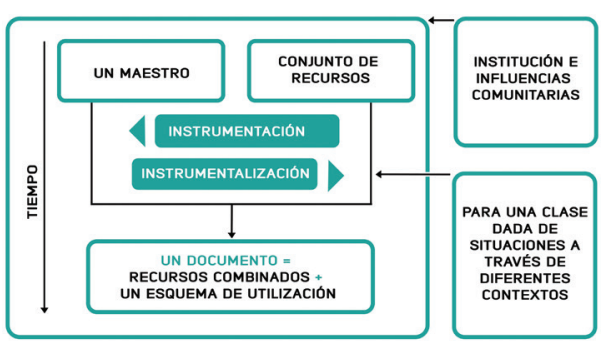

Figura 6. Modelización de una génesis documental (Gueudet \& Trouche 2012, p. 26).

La importancia del trabajo documental de los profesores nos ha llevado a desarrollar un enfoque que amplía el enfoque instrumental y, que además, se basa en otras investigaciones. Éstas se refieren, en particular, a la ingeniería documental (Pédauque, 2006), que destacó los trastornos vinculados a la digitalización, y al estudio del curriculum material (Remillard, 2005) que considera las interacciones entre profesores y documentación escolar. 
El maestro interactúa con los recursos cuya extensión excede a la de los artefactos. Consideramos que, durante una génesis documental, un conjunto de recursos da origen a un documento para una clase de situaciones (figura 6). Este término, inspirado en la ingeniería documental (Pédauque, 2006), designa así a un concepto que generaliza el de instrumento. El trabajo documental del docente es el motor de una génesis documental, que desarrolla conjuntamente un nuevo recurso (compuesto por un conjunto de recursos seleccionados, modificados, recombinados) y un esquema de uso de este recurso. Podemos representar este proceso, de manera muy simplificada, por la ecuación: Documento = Recursos recombinados + Esquema de uso.

Un esquema contiene fundamentalmente normas de acción e invariantes operatorios, que representan aquí los conocimientos profesionales de los docentes. No nos referimos a una categorización de dichos conocimientos, sino a designar con este término a todos los conocimientos susceptibles de intervenir en la actividad profesional de los docentes. Estos conocimientos pueden ser matemáticos, o referirse a los tipos de tareas que se ofrecen a los estudiantes, las dificultades para anticipar, etc. La noción de invariante es compatible con el aspecto dinámico de las génesis: frente a recursos nuevos y a una nueva clase de situaciones, el profesor recurre a su repertorio de invariantes; éstos son asociados, modificados, generando nuevos invariantes. En consecuencia, la interacción con los recursos es esencial para la evolución de los conocimientos de los docentes. Los recursos que trabaja un profesor son recursos vivos, continuamente renovados, y el análisis de su desarrollo profesional puede basarse en el análisis de la trayectoria de los recursos que pone en acción.

Las modelizaciones presentadas en las figuras 5 y 6 parecen muy similares, sin embargo, difieren profundamente. El enfoque instrumental fue desarrollado principalmente para entender los efectos de la integración de un nuevo artefacto en la actividad de un estudiante o maestro. El enfoque documental pretende ser un enfoque holístico, el cual toma en cuenta al conjunto de recursos, antiguos, recientes o nuevos que entran en juego en el trabajo del profesor. En este contexto, los procesos de instrumentación e instrumentalización adquieren una nueva dimensión: para la instrumentación, ya no es sólo un artefacto, sino un vasto conjunto de recursos que van a contribuir a configurar la actividad didáctica de docente; para la instrumentalización, la actividad creativa del profesor es mucho más visible cuando adapta para adoptar los recursos, que cuando se apropia de un artefacto dado. Esta diversidad de recursos ha llevado a la introducción de nuevos conceptos, dando especial importancia al concepto de sistema. 


\subsection{SISTEMAS DE RECURSOS, SISTEMAS DOCUMENTALES Y SISTEMAS DE ACTIVIDAD}

En un primer momento se puede considerar que los documentos de un profesor se articulan de acuerdo con la estructuración institucional del contenido matemático de la cátedra. Sin embargo, la observación de varios docentes muestra de entrada, diferencias significativas de uno a otro, que indican la necesidad de ir más allá de este primer nivel explicativo. Dentro del marco del enfoque instrumental, Rabardel y Bowley (2005) consideran sistemas instrumentales organizados, cuya estructura está relacionada con aquella de la actividad profesional de los sujetos. Más allá de los tipos de situaciones, que dan sustancia al desarrollo del mismo esquema, Rabardel y Bowley proponen una agrupación de situaciones de actividad profesional en familias de actividad, conjuntos de tipos de situaciones que corresponden a un mismo tipo de propósito general de la acción. Retomando esta perspectiva, llamamos sistema de actividad al conjunto de familias de actividad de un sujeto (por ejemplo: preparar las clases, corregir exámenes, o más aún implementar recursos en el aula). Consideramos, al igual que Rabardel y Bowley, que los documentos de un profesor se articulan en sistemas documentales, en relación con sus sistemas de actividad. Se trata de una elección teórica, llevada a cabo a priori en consonancia con el enfoque instrumental y con la importancia dada a los conocimientos profesionales de los docentes.

El sistema de recursos del profesor constituye la parte de "recursos" de su sistema documental (es decir, la parte esquemas de los documentos). Desde la perspectiva que adoptamos, el sistema documental está compuesto por el sistema de recursos y de conocimientos profesionales asociados. Todo esto se articula con el sistema de la actividad docente. En los desarrollos ulteriores del enfoque documental, en lugar de una lógica top-down, que se centra en primera instancia en el sistema documental y, después en el sistema de recursos del profesor, hemos desarrollado una lógica bottom-up, que se enfoca en primer lugar en el sistema de recursos del maestro, antes de intentar inferir elementos de su sistema documental (véase por ejemplo Pepin et al. 2016). El sistema de recursos del profesor es entonces la unidad de análisis relevante para entender su actividad, en particular la integración, o la integrabilidad, de un nuevo recurso que le es propuesto.

El lector interesado podrá encontrar ejemplos de estudios de génesis documentales, de procesos de instrumentación e instrumentalización en las entradas de la Encyclopedia of Mathematics Education recientemente publicada (Trouche, 
2018b, Trouche, 2018c, Trouche, Gueudet \& Pepin, 2018). En la siguiente sección, nos concentraremos en las diferentes formas que puede tomar el trabajo docente con recursos, desde una perspectiva de capacitación.

\section{UN RECURSO SUSCEPTIBLE DE ESTIMULAR LA ACTIVIDAD MATEMÁTICA DE DOCENTES Y DE ESTUDIANTES}

Esta sección puede considerarse como un paréntesis o un respiro matemático en este artículo. Se trata de proponer un problema matemático, susceptible de regenerar la actividad matemática de un estudiante o de un maestro. Veremos en las siguientes secciones ( 4 y 5) cómo se puede pensar este problema desde la perspectiva de una integración al sistema de recursos de un docente. Presentamos en primer lugar, el problema en sí y luego damos algunos resultados derivados de un breve brainstorming (lluvia de ideas) de estudiantes, profesores e investigadores que se vieron confrontados con este problema.

\subsection{El PROBlema 'TRAZOS DE Bicicleta'}

Geometry and the Imagination (Geometría y la imaginación) es un libro de David Hilbert y Stephan Cohn-Vossen, basado en una serie de conferencias que Hilbert realizó en el invierno de 1920-21, publicado originalmente en 1952. John Conway, Peter Doyle, Jane Gilman, Bill Thurston retomaron este título para un curso impartido en el Centro de Geometría de la Universidad de Minnesota en junio de 1991 (https://math.dartmouth.edu/ doyle/docs/gi/gi.pdf). El problema de los "trazos de bicicleta" (ver ventana 1) proviene de este curso. 


\section{Ventana 1 \\ Trazos de bicicleta}

C. Dennis Thron llamó la atención sobre el siguiente pasaje de The Adventure of the Priory School (La aventura del Colegio Priory), de Sir Arthur Conan Doyle:

- "Este trazo, como puede usted notar, fue hecho por un ciclista que iba desde la dirección de la escuela".

- ¿O hacia ella?

- No, no, mi querido Watson. La impresión de hundimiento más profunda es, por supuesto, la de la llanta trasera, sobre la cual descansa el peso. Nota usted las diferentes partes por donde la atravesó y borró la marca más superficial de la delantera. Sin duda, se estaba alejando de la escuela".

Problemas

1. Comente este pasaje. ¿Sabe Holmes de qué está hablando?

2. Intente determinar la dirección de desplazamiento de los trazos de bicicleta imaginarios en la siguiente figura.

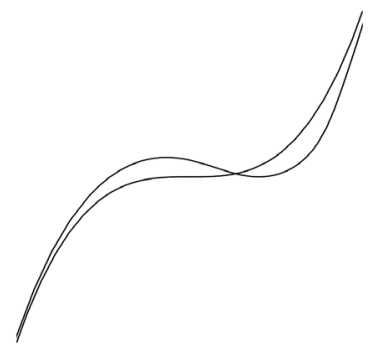

3. Trate de dibujar algunos trazos propios de bicicleta imaginarios. No necesitas una computadora para esto; sólo una idea de cuál es la relación entre el trazo de la llanta delantera y el trazo de la llanta trasera. ¿Qué tan buenos creen que son sus trazos simulados?

4. Salga y observe algunos trazos de bicicleta en la naturaleza. ¿Puede decir en qué dirección iba la bicicleta? No pierda de vista los trazos de la bicicleta y practique hasta que pueda determinar la dirección del desplazamiento de forma rápida y precisa.

\subsection{AlgunAS RESPUESTAS DERIVADAS DE UN CORTO BRAINSTORMING (LLUVIA DE IDEAS)}

Durante el seminario impartido en el Instituto Freudenthal (Trouche, 2018a), propuse una breve lluvia de ideas a los participantes, orquestando el trabajo de la siguiente manera: 
La figura 1 se mostró en la pantalla y en una hoja en línea compartida, con la pregunta '¿Podría proponer una idea para introducir el problema (centrándose en una característica determinada del dibujo y/o utilizando un artefacto dado, y/o un marco matemático dado, o ...)?

- En pequeños grupos de trabajo (4 personas cada uno), con al menos una computadora portátil conectada -por grupo-, debiendo anotar sus ideas en la hoja compartida en línea.

- 30 minutos de reflexión.

Los seis grupos propusieron los siguientes elementos de respuestas (retomamos exactamente sus palabras):

- Grupo 1: "Creamos un dibujo con una memoria USB y unos lápices frontal y posterior que actuaron como bicicleta creando curvas mientras la movíamos sobre un papel. Este experimento nos permitió suponer que la bicicleta se mueve "hacia arriba", ya que el punto de intersección sucede "más adelante". La llanta delantera hace las curvas más grandes".

- Grupo 2: "Determine primero cuál es la llanta delantera mirando la intersección y la curvatura (curva más grande)".

- Grupo 3: "La curvatura en la esquina superior derecha es menos nítida. Parece una corrección. ¿Podemos ver la profundidad de la punta? ¿Tiene simetría central? Más o menos"...

- Grupo 4: "Dos Ilantas. La llanta delantera puede tener curvas más nítidas. La distancia entre los puntos de las llantas es constante (independientemente de la orientación de la llanta delantera). Problema: no sabemos el tamaño de la bicicleta. Solución-sugerencia: enfoque iterativo del uso de un modelo de bicicleta de longitud variable, ver si hay una longitud donde la distancia entre los puntos es constante".

- Grupo 5: "Giramos la laptop y descubrimos una diferencia en las curvas. Más curvada $\rightarrow$ Ilanta delantera. La distancia entre la parte trasera y la delantera es constante $\rightarrow$ no se puede usar eso (triángulo fijo). ¿Tendría importancia la superficie entre las curvas? ¿Derivada?"

- Grupo 6: “¿Qué trazo corresponde a qué llanta (delantera o trasera)? Vemos la simetría central: es difícil decidir en qué dirección va la bicicleta. ¿Lo investigamos de manera teórica o empírica? Ponemos a alguien a andar en bicicleta... ¿̇ usamos nuestra memoria muscular? 
Estas respuestas, reunidas en la hoja compartida en línea, sólo muestran una parte de la actividad de los participantes: no tenemos acceso a todos los pequeños dibujos realizados a lo largo del brainstorming, ni a los gestos producidos, por ejemplo, con la memoria USB actuando como la bicicleta... Sin embargo, podemos imaginar la riqueza y variedad de esta actividad, destacando el potencial de este problema.

La actividad aquí está muy limitada por el tiempo (isólo 30 minutos!). Está dirigida a personas que más bien son expertas en este tipo de problemas (estudiantes de doctorado, docentes e investigadores de un Instituto, el Institut Freudenthal, dedicado a la reflexión sobre la educación matemática analizando "problemas realistas" (ver por ejemplo Van den Heuvel-Panhuizen, 2003). ¿Cómo pensar el análisis de este problema en un tiempo más largo, con la perspectiva de una integración real de un nuevo recurso en el sistema de recursos de los estudiantes o de los profesores 'comunes'? Esto es lo que vamos a estudiar en la próxima sección.

\section{UN PROBLEMA INTEGRADO EN 2002 EN EL CONTEXTO DE UN PROGRAMA AVANZADO DE FORMACIÓN DE MAESTROS EN FRANCIA}

Veremos en esta sección el análisis del recurso "Trazos de bicicleta" en un contexto de capacitación continua de docentes. En una primera parte, presentamos el dispositivo de formación de los maestros en cuestión, el SFoDEM; en una segunda parte, presentamos el modelo de recurso derivado del trabajo colaborativo de los profesores involucrados en el SFoDEM; por último, presentamos la aplicación del recurso "Trazos de bicicleta" al modelo SFoDEM.

\subsection{EL SFODEM, UN DISPOSITIVO DE FORMACIÓN DOCENTE AMBICIOSO, PENSADO A LARGO PLAZO}

SFoDEM (en Francés: Suivi de formation à distance d'enseignants de mathématiques / seguimiento de la capacitación de docentes de matemáticas a distancia) fue diseñado e implementado en Francia, del 2000 al 2006, por el IREM (Instituto de Investigación sobre la Enseñanza de Matemáticas) de Montpellier, con el objetivo de ayudar a los profesores de matemáticas a integrar en sus prácticas pedagógicas las TIC (Guin y Trouche, 2005). Se basaba en cinco hipótesis principales: cambiar las prácticas requiere de tiempo (por lo 
tanto, la duración de la capacitación: 6 años); requiere de un apoyo continuo (por consiguiente, el uso de una plataforma en línea para apoyar las interacciones de los maestros entre dos reuniones en persona); requiere de un trabajo de diseño conjunto que asocie docentes y formadores de docentes, para renovar los recursos que integran las TIC y que se implementan en las clases; requiere de la articulación de las fases de diseño colaborativo, de implementación en el aula, de pensamiento reflexivo y de revisión de recursos. Debe tener en cuenta el contexto de la formación (tanto tecnológica como matemática). Parece claro que el SFoDEM fue una incubadora para el enfoque documental en la didáctica que se desarrollaría más adelante y, se basaría fundamentalmente en la idea de una evolución conjunta de los recursos y del conocimiento de los docentes.

Se eligieron cinco temas de capacitación (transición de marcos numéricos a algebraicos y TIC; cálculo y calculadoras gráficas y simbólicas; software de geometría 2D y geometría dinámica, simulación de experiencias aleatorias y resolución cooperativa de problemas por Internet). Cada tema tenía una libertad relativa para desarrollar sus recursos de acuerdo con un modelo que se ajustaba perfectamente a sus propios contextos tecnológicos y matemáticos, y un comité de capacitación que reunía a todos los participantes, trató de darle sentido a las diferentes experiencias hacia posibles (al menos parcialmente) modelos comunes, tanto para la organización de las interacciones como para los propios recursos. Las conclusiones principales de estas experiencias se presentan en un CDRom (Guin, Joab \& Trouche, 2008).

Una de estas conclusiones fue que los recursos iniciales proporcionados por los capacitadores, a menudo recursos expertos, eran demasiado complejos para una experimentación por parte de los alumnos en su propia clase. Por lo tanto, hubo una evolución hacia recursos más simples, más fáciles de implementar y hacia talleres virtuales de estudiantes creando recursos que provenían de sus propios sistemas de recursos, llamados "gérmenes de recursos". Esta evolución puede considerarse como una evolución desde un enfoque de arriba hacia abajo hacia un enfoque de abajo hacia arriba. Otra conclusión fue el interés por tener un modelo común que permitiera compartir los recursos, un modelo que constituyera un apoyo a los docentes, tanto para el diseño de nuevos recursos como para sus usos. 


\subsection{EL MODELO SFODEM, SURGIDO DEL TRABAJO COLABORATIVO DE LOS DOCENTES Y DEL CRUZAMIENTO DE LOS DIFERENTES TEMAS DE FORMACIÓN}

El trabajo a distancia y los requisitos de comunicación han conducido a realizar una aclaración de las acciones que acompañan el diseño e implementación de cada recurso (Gueudet \& Trouche, 2008). De esta manera, en el grupo dedicado a la integración de los softwares de geometría dinámica, el trabajo documental común pasa por varias etapas sucesivas: la elección de un problema matemático, la reflexión sobre la posible contribución de un software de geometría en el análisis de esta situación en el aula, la elaboración de una "ficha" para los alumnos, una concepción a priori de los escenarios de uso (es decir, de propuestas para orquestaciones instrumentales), la implementación efectiva del recurso en cada una de las clases, la discusión colectiva sobre los efectos de esta implementación, la elaboración de una ficha destinada a otro profesor (proponiendo posibles variables para el escenario y advirtiendo posibles dificultades), la reflexión sobre los vínculos que se establecerán entre las diferentes fichas digitalizadas, la reflexión sobre la indexación de este recurso. Asimismo, se observa el desarrollo de registros comunes para cada tema de capacitación.

Este desarrollo de un registro en cada grupo va de la mano con la creación de un modelo común de recursos, en tanto que resultado de un proceso de modelización del trabajo documental: lo vimos en el grupo geometría, a través del surgimiento de escenarios de uso, de informes de experimentación. Este modelo ha evolucionado de forma iterativa, a través de idas y vueltas entre los recursos existentes en cada grupo y los recursos encontrados entre las necesidades de cada tema y las necesidades de todo el SFoDEM. La figura 7 muestra las etapas principales de esta evolución. Inicialmente (en el año 2000), coexistían diferentes modelos, dependiendo de los grupos (por ejemplo, una breve descripción del recurso, una ficha para el alumno y una ficha para el docente brindando algunos elementos de reflexión sobre el problema planteado). Gradualmente, los modelos iniciales se enriquecieron y la confrontación de los diferentes modelos permitió el establecimiento de un modelo común, por ejemplo, la incorporación de una ficha seguimiento del trabajo de los estudiantes, que apareció en el grupo resolución de problemas que quería integrar en cada recurso algunas narrativas de investigación de los estudiantes y no lograba hacerlo en el informe de experimentación, el cual se utilizaba más para incorporar las observaciones del docente. Esta inserción se discute en los otros grupos del SFoDEM, quienes lo van a adaptar a nuevos usos (la integración de capturas de pantalla en el recurso, o de capturas 
de alumnos que corresponden a momentos críticos del trabajo en el aula, como resultado y soporte de una reflexión didáctica).

La última adición a la estructura es el CV (curriculum vitae del recurso). El cual permite incluir en el recurso su propia historia, sus autores sucesivos y las motivaciones de sus evoluciones. El recurso está vivo, y cada nuevo usuario, al apropiárselo, se constituye como un nuevo eslabón de una cadena de usuarios/ autores. Los archivos satélite son elementos comunes de varios recursos: puede tratarse de elementos de uso de un software, o de elementos teóricos producidos a partir de la reflexión de uno de los temas de formación.
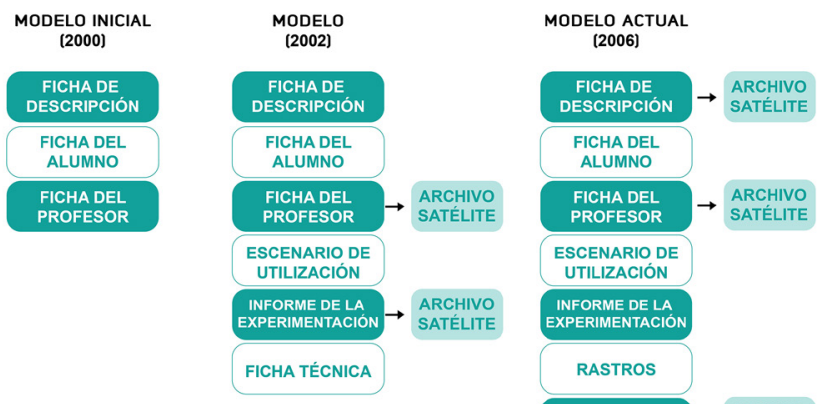

FICHA TÉCNICA $\rightarrow$ ARCHIVO

Figura 7. La evolución del modelo de recursos SFoDEM (Gueudet \& Trouche, 2008, p. 25).

Este modelo proporciona un marco general para el diseño. Le proporciona un tipo de objetivo a los autores de recursos, pero, prácticamente, la estructura de los recursos diseñados puede variar, como lo muestra la mediateca de recursos SFoDEM (Guin, Joab \& Trouche, 2006), dependiendo del grado de madurez del recurso concebido o de la experiencia de los diseñadores.

\subsection{El PROBLEMA DE LAS “TRAZOS DE BICICLETA” DIGERIDO POR EL SFODEM}

Un investigador que participó en el grupo de capacitación 'Cooperative problem solving via Internet' (resolución en común de problemas vía internet), en 2005, presentó el problema 'Bicycle tracks' hacia el final de la existencia del dispositivo SFoDEM. En consecuencia, el grupo no tuvo tiempo de desarrollar todo el recurso de acuerdo con el modelo. Sólo desarrolló tres elementos de este modelo: una ficha de identificación, una ficha estudiante y una ficha docente. 
Encontraremos (ventana 2) la ficha docente, que restituye la experiencia del grupo de docentes que trabajaron este recurso: dos horas en el grupo de formación, intercambiando, en pequeños grupos profesores y formadores, resolviendo el problema para ellos mismos, posteriormente en sus clases, durante varias sesiones de trabajo, y luego nuevamente en el grupo de formación para realizar una revisión crítica de las interacciones con los estudiantes. El grupo utiliza internet en dos niveles: para intercambios entre profesores y para intercambios entre clases. Lo cual corresponde aproximadamente a unas diez horas de trabajo para cada docente, sobre este recurso (que se compararán con los 30 minutos de reflexión movilizados en el seminario del Instituto Freudenthal $(3,3)$.

\section{Ventana 2 \\ Trazos de bicicleta': La ficha docente diseñada por un grupo del SFoDEM}

http://www.math.univ-montp2.fr/sfodem/BibliothequeSfodem/sfodem HTML/Chap1/th5r12 fp1.html

El interés principal de este problema es el de la modelización y el de la toma de decisiones que dependen de ella. La pregunta en sí misma a menudo plantea grandes problemas para los estudiantes.

- ¿ ¿Por qué hacerse esta pregunta? El problema puede presentarse como un acertijo policíaco, con referencia a Sherlock Holmes.

- $\quad$ ¿Este trazo fue hecho por una sola bicicleta o por dos? ¿No podrían ser dos, ya que hay dos trazos? A los estudiantes les cuesta trabajo concebir la naturaleza del trazo que puede dejar una bicicleta en el suelo. Para ayudarles, se les puede sugerir que se imaginen empujando una bicicleta por el suelo, observando cómo se mueven las llantas; ¿Qué tipo de trazo van a ver? En particular, ¿el trazo de la llanta trasera cubre siempre el de la delantera?

- ¿ ¿La velocidad tiene un papel específico?

- ¿ ¿Sabemos si el terreno sube o baja?

- $\quad$ ¿Son posibles los derrapes? Esta pregunta es importante porque se trata de elegir de manera razonable y ver qué trabajo se puede hacer a partir de esa elección. A priori, nada impide imaginarse que la bicicleta derrapó en el lugar del trazo... Si los derrapes están permitidos, uno podría pensar que «todo podría haber sucedido» (pensar por ejemplo en un equilibrista o en un virtuoso de la bicicleta acrobática: sin duda pueden hacer trazos muy extraños en el suelo) y por lo tanto el problema no se puede resolver (no se dispone de suficientes datos). Sin embargo, frente a la elección de «derrape o no derrape», se puede hacer sentir a los estudiantes que hay dos problemas, uno de los cuales está contenido en el otro, y que en esta circunstancia es más fácil resolver el caso en el cual la limitante es más grande. De hecho, a posteriori se podrá justificar esta opción, puesto que el análisis de los trazos demuestra efectivamente que una bicicleta podría hacerlos sin necesidad de llevar a cabo ningún derrape; Se concluye que, de haber habido derrapes, éstos serían probablemente irrelevantes. Otro problema de 
modelización para los más exigentes: puesto que el eje del manubrio de una bicicleta no es vertical, la distancia entre los puntos de contacto de las llantas con el suelo se modifica ligeramente cuando la bicicleta gira... De la misma manera, tal vez podamos pasar por alto este punto en un primer momento y ver si llegamos a algo interesante.

Una vez que se admite que son los trazos dejados por una sola bicicleta durante el mismo trayecto, los estudiantes tratan generalmente de distinguir los trazos de las llantas delanteras y traseras. Con frecuencia surgen dos conjeturas naturales:

- La llanta trasera es la que recorre menos camino;

- La llanta delantera es la que tiene la curvatura más grande;

Otros dos elementos del trazo llaman la atención:

- $\quad$ El punto de cruzamiento: ¿Cómo analizarlo? ¿Provee alguna información?

- Los puntos de inflexión de las curvas: muestran el «momento» en el cual la bicicleta cambió de dirección. ¿̇legan de manera simultánea?

Para ayudar con la resolución, se puede hacer la siguiente pregunta, que puede parecer extraña: «¿cuál era el tamaño de la bicicleta a la escala del dibujo?» La idea es sugerir a los alumnos que representen la bicicleta en diferentes puntos del trayecto, y hacerles descubrir que uno de los dos sentidos es imposible si se quiere que la bicicleta tenga un tamaño constante.

Algunas posibles extensiones:

- ¿ ¿Dos bicicletas de diferente tamaño pueden dejar trazos idénticos?

- ¿ ¿Es posible que los mismos trazos puedan recorrerse en una y otra dirección?

La clave del problema radica en el hecho de que en todo momento las llantas de la bicicleta son tangentes a los trazos que dejan en el suelo. Desde este punto de vista, la llanta trasera es la más interesante porque siempre se encuentra en el eje de la bicicleta (no gira). Si tomamos un punto del trazo de la llanta trasera (AV del dibujo que está a continuación) y extendiéramos su tangente en el sentido de la trayectoria, esta tangente debe cortar el trazo de la llanta delantera en un punto (AV del dibujo que está a continuación) de modo que la distancia entre AR y AV sea constante. En la figura propuesta, este análisis permite identificar los trazos (llanta trasera y llanta delantera) y determinar la dirección de recorrido.

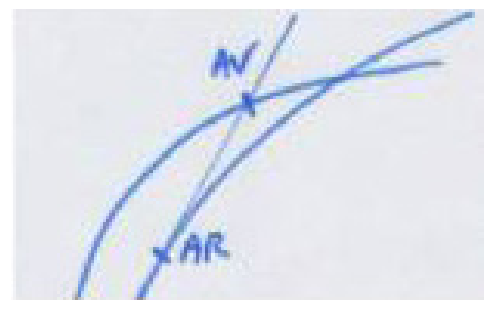


La lectura de esta ficha docente, producida a partir de la experiencia de los profesores involucrados en el grupo, merece varias observaciones.

En primer lugar, demuestra la riqueza de la ficha docente, ya que comparte la experiencia de la colaboración de los profesores y la cooperación de los alumnos en las clases. La variedad de preguntas planteadas y la variedad de elementos de observación que podrían ser relevantes, parecen además realmente útiles para que un profesor, que no ha vivido directamente la capacitación, tenga la capacidad de aplicar el problema en su clase.

En segundo lugar, resulta sorprendente el hecho de que nadie sugiere llevar a cabo el experimento por sí mismo, yendo con una bicicleta sobre la arena...; nadie sugiere tampoco usar un artefacto para modelizar la trayectoria de una bicicleta (como la memoria USB en el seminario del Instituto Freudenthal), o un software de geometría, pese a que la capacitación en los TIC constituye un punto sensible del dispositivo SFoDEM. Finalmente, (mientras que Internet es una de las herramientas de este tema de formación), nadie sugiere usar el Internet para encontrar recursos adicionales para ayudar a esclarecer el problema. Las herramientas sugeridas son las utilizadas para generar la representación gráfica "a mano" de los trazos de la bicicleta. Veremos en la siguiente sección que, 13 años más tarde y en otro contexto, los recursos movilizados son muy diferentes.

Por último, tenemos aquí un buen ejemplo de instrumentalización de un recurso inicial, el problema 'Bicycle tracks', adaptado por los miembros del grupo para ser integrado en sus sistemas de recursos. Cómo actuó la instrumentación para cada uno de ellos, es decir, cómo condujo a cambiar su propia actividad en el aula la integración de este nuevo recurso: el CDROM (Guin, Joab \& Trouche, 2008) ofrece testimonios de actores que ponen en evidencia la influencia del trabajo documental colectivo por un tiempo bastante largo, sobre el trabajo documental individual de cada actor.

\section{UN PROBLEMA INTEGRADO EN LA LABOR DE UN CICLO DE SEMINARIOS EN CINVESTAV (MÉXICO) EN ABRIL DE 2017}

The problem 'Bicycle tracks' was proposed in 2017, 12 years after the experience presented in the previous section, in the context of a scientific stay, answering to the invitation of Luis Moreno and Armando Cuevas, in the CINVESTAV department of mathematics education (Mexico). En esta sección presentamos la orquestación de la investigación en torno a este problema, seguido de dos hechos 
primordiales: primero, la aportación de los softwares de geometría dinámica y después, el requerimiento del Internet.

\subsection{ORQUESTACIÓN DE LA INVESTIGACIÓN}

Esta estadía se organizó en torno a un seminario de trabajo mensual, reuniendo a estudiantes de doctorado e investigadores del Departamento. La sesión del seminario del 7 de abril se dedicó a la presentación del libro Tools and mathematics, instruments for learning (Monaghan, Trouche, \& Borwein, 2016). Intitulado Explorando, compartiendo experiencia y experimentando - una travesía inspiradora en la (educación) matemática, el seminario proponía el análisis de diversos problemas, siendo el último el problema de 'Bicycle track's (figura 8). Se invitó a los miembros del seminario a reflexionar sobre este problema, individualmente o en grupos, y a compartir las soluciones, incluso parciales, en un espacio compartido en línea.

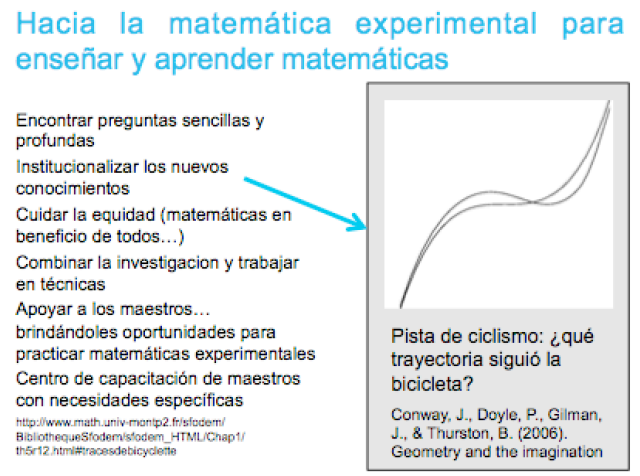

Figura 8. Presentación del problema en el seminario del 7 de abril de 2017.

La reflexión se retomó en un seminario organizado conjuntamente con Ana Isabel Sacristán, el 24 de abril de 2017, titulado Estimular la creatividad para el aprendizaje y enseñanza de las matemáticas. Ahí, se invitó a los participantes a analizar el problema en pequeños grupos durante 30 minutos y luego a insertar las respuestas posibles en una tabla, que a su vez permitiera confrontar los diferentes enfoques (figura 9). Luego de una discusión general, destacando los puntos de apoyo para la resolución, se tomaron capturas de pantalla de la 
tabla, almacenadas en el espacio compartido en línea, de manera que todos pudieran continuar con la reflexión.

De esta manera, la resolución del problema se realizó mediante etapas sucesivas combinando la discusión grupal, la reflexión personal y los intercambios por correo electrónico entre estudiantes e investigadores. ¿Qué debe conservarse de esos intercambios, tanto para la economía de la resolución como para el desarrollo de los sistemas de recursos de los actores interesados?

\subsection{LA CONTRIBUCIÓN DE LOS SOFTWARES DE GEOMETRÍA}

La primera diferencia notable es la movilización de los softwares de geometría dinámica, requeridos naturalmente, como herramientas de resolución de problemas (aun cuando estas herramientas no habían aparecido hace 12 años). Como vemos al principio de la resolución de un estudiante (ventana 3), aprovechar un software de geometría dinámica equivale a expresar matemáticamente las limitantes de la figura, lo que es, de hecho, emprender un proceso de construcción y prueba.
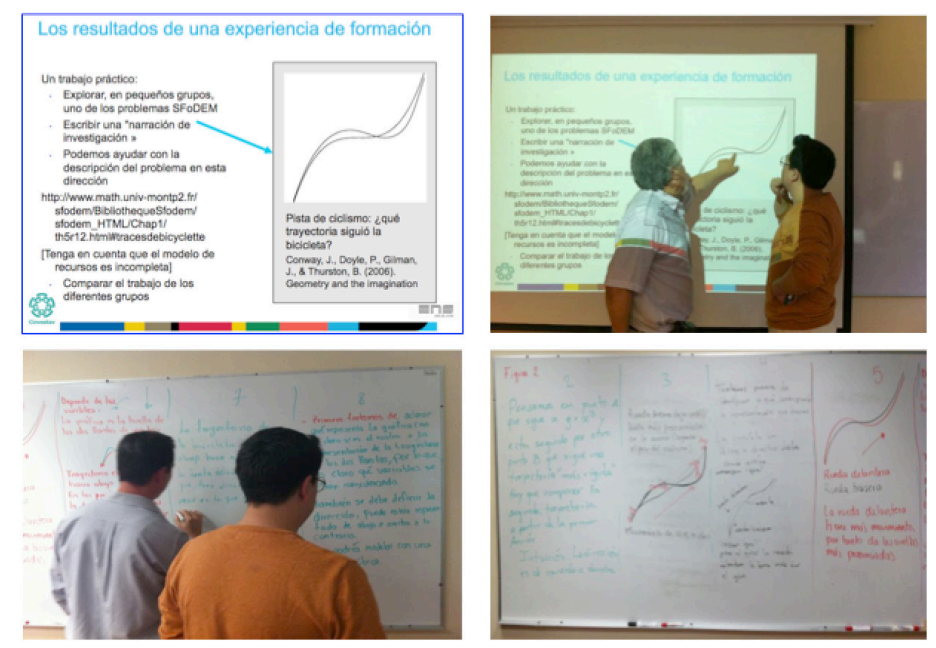

Figura 9. La organización de la reflexión en el seminario del 24 de abril de 2017. 


\section{Ventana 3 \\ Movimiento de las llantas de la bicicleta \\ Juan Jesús Gutiérrez García - juan.gutierrez@cinvestav.mx}

\section{Resumen}

Se describe cómo aproximar el trazo que dejan las llantas de una bicicleta sobre el suelo, utilizando GeoGebra. Para ello se aproxima el movimiento de la llanta trasera de la bicicleta suponiendo que la llanta delantera sigue una curva definida por una función. El problema original es propuesto por el Dr. Trouche en una de sus conferencias.

\section{Descripción de la aproximación}

Se realizan las suposiciones siguientes, la longitud de la bicicleta es conocida y se llama Longb, la trayectoria que sigue la llanta delantera es una función que llamamos trayx y definimos como x3 pero se podrá cambiar para realizar diferentes recorridos. El punto de contacto de la llanta delantera con el piso se llamará A y se definirá como un punto sobre la función tray $(\mathrm{x})$. Con estos elementos definimos que el punto de contacto de la llanta trasera con el piso se llamará B y estará a una distancia Longb del punto $\mathrm{A}$, es decir sobre una circunferencia de radio Longb y centrada en A, esto nos lleva a una configuración como la que se muestra en la figura a.

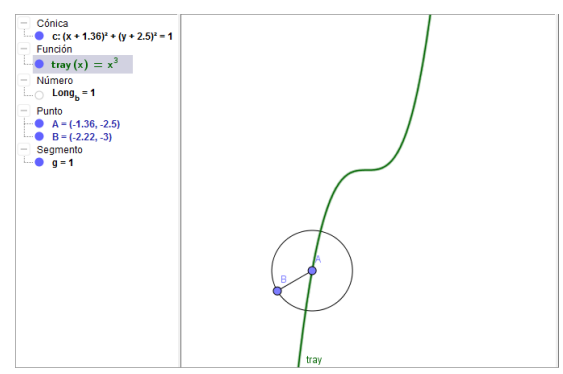

Figura a. Configuración inicial para aproximar el movimiento del punto B

El segmento marcado como g describe la posición inicial de la bicicleta antes de comenzar a avanzar, y como el punto B es libre se puede comenzar desde cualquier posición posible.

Ahora, cuando el punto A se desplace por la trayectoria arrastrará al punto B. Si suponemos un movimiento pequeño del punto A entonces el punto B se desplazará sobre la dirección del segmento BA pero manteniendo fija la distancia entre la nueva posición de A y la nueva posición de B. En la figura b se ejemplifica este movimiento, señalando a los puntos nuevos como A' y $\mathrm{B}^{\prime}$, además en esta figura el desplazamiento del punto A es bastante grande y marcado como paso para ejemplificar cómo calcular el punto B'.

El punto $\mathrm{A}^{\prime}$ se define como el punto sobre la trayectoria que corresponde a un desplazamiento paso sobre el eje de las $x$, esto es:

$A^{\prime}=(x A+p a s o, \operatorname{tray}(x A+p a s o)$ 
Con centro en el punto A' se traza una circunferencia, punteada en la figura b, del radio igual a la longitud de la bicicleta, Longb. Entonces el punto B' será la intersección entre el segmento BA y la circunferencia punteada. [...]

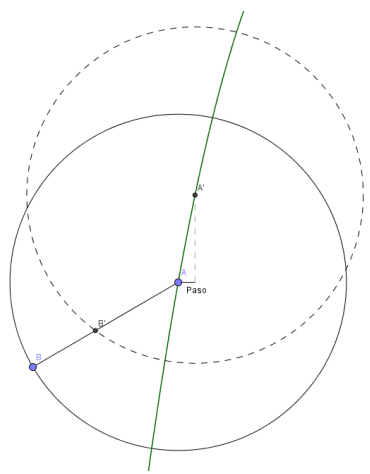

Figura b. Aproximar el movimiento del punto B al moverse el punto A.

El punto B' aproximará mejor el movimiento de la llanta trasera de la bicicleta si el valor de paso es pequeño. Si ésta variable toma un valor "muy grande" es posible que el círculo punteado no intercepte al segmento BA. [...]

De hecho, es el principio de la resolución del problema, pero sólo toma en cuenta una de las limitantes: la distancia constante entre los puntos de contacto de las dos llantas con el suelo. La limitante de las tangentes no se ve: lo cual implicaría pensar la construcción a partir de la llanta trasera y no de la llanta delantera, lo cual va a destacar más adelante las discusiones del seminario, apoyándose también en las contribuciones de los recursos considerados en Internet.

\subsection{LA CONTRIBUCIÓN DEL INTERNET}

Una segunda diferencia significativa entre las exploraciones matemáticas de 2005 y las de 2017 es la contribución de otros recursos a los propuestos por el problema mismo, en particular de los recursos de Internet.

El primer recurso fue propuesto por Luis Moreno, director del Departamento de Matemática Educativa de CINVESTAV, al señalar que el problema también había sido estudiado por Tom Apostol y Mamikon Mnatsakanian en su libro 
(2012) sobre los Nuevos horizontes geométricos y al producir, al mismo tiempo, una foto en la que vemos al mismo Mamikon Mnatsakanian, en bicicleta, dejando los famosos trazos en la arena (figura 10).

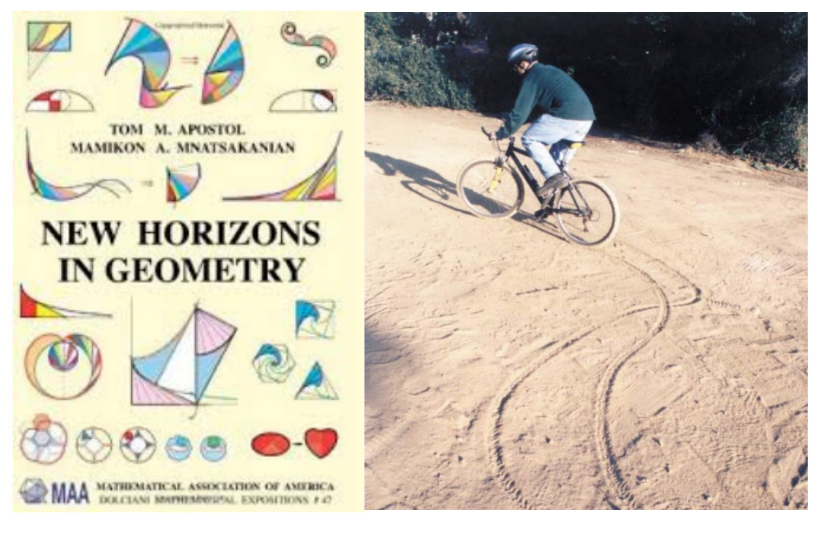

Figura 10. Mamikon Mnatsakanian coautor de la obra, aparece experimentando él mismo las trazas de una bicicleta en la arena.

De hecho, lo que se está desarrollando involucra una dialéctica entre 'construir soluciones' y 'buscar soluciones preconcebidas'. De este modo, la construcción de una solución en GeoGebra está respaldada por una búsqueda de simulación de los trazos de una bicicleta sobre la arena, que se puede ver en esta dirección http://www.thedudeminds.net/?p=5636 (figura 11)

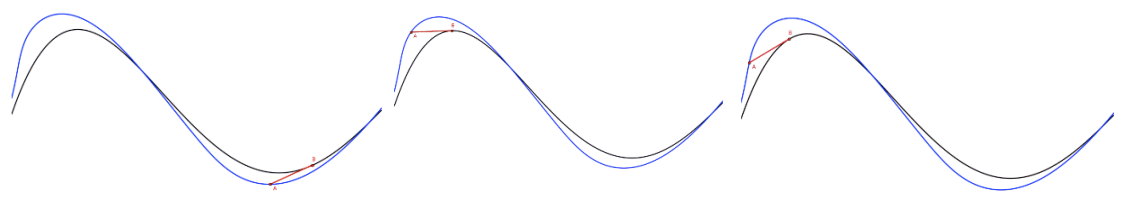

Figura 11. Una simulación de la trayectoria de la bicicleta y del trazo de las llantas http://www.thedudeminds.net/?p=5636

De hecho, el análisis de GeoGebra planteaba dos preguntas: ¿Cómo obtener representaciones gráficas de trazos de llantas de bicicleta (aspecto geométrico)? ¿Cómo obtener las ecuaciones de las curvas correspondientes (aspecto algebraico)? El análisis en Internet permite obtener respuestas también para la segunda pregunta (figura 
12). Obtener estas respuestas supone formular bien las preguntas, utilizando palabras clave que se acumulan a medida que nos adentramos en el problema (bicycle tracks, Sherlock Holmes, Apostol...), jugando también con varios idiomas (formular las consultas de Internet en inglés, francés, español...).

Por lo tanto en 2017 hay una dinámica de la resolución muy diferente de la de 2005, y esta metamorfosis está ligada, profundamente, al desarrollo de Internet: en 2005 se aborda la resolución de un problema movilizando su propio sistema de recursos y jugando con la sinergia entre su propio sistema de recursos y los de sus colegas, en la dinámica de la cooperación (por ejemplo, dentro de los dispositivos de formación). En 2017, la resolución de un problema se aborda extendiendo el sistema de recursos por medio de la integración como con un rompecabezas, piezas sucesivas que permiten esclarecer las preguntas planteadas. Por lo tanto, el sistema de recursos de los estudiantes y los profesores debe considerarse como sistema dinámico abierto cuya (re)construcción sigue sugiriendo el planteamiento de complejas preguntas teóricas.

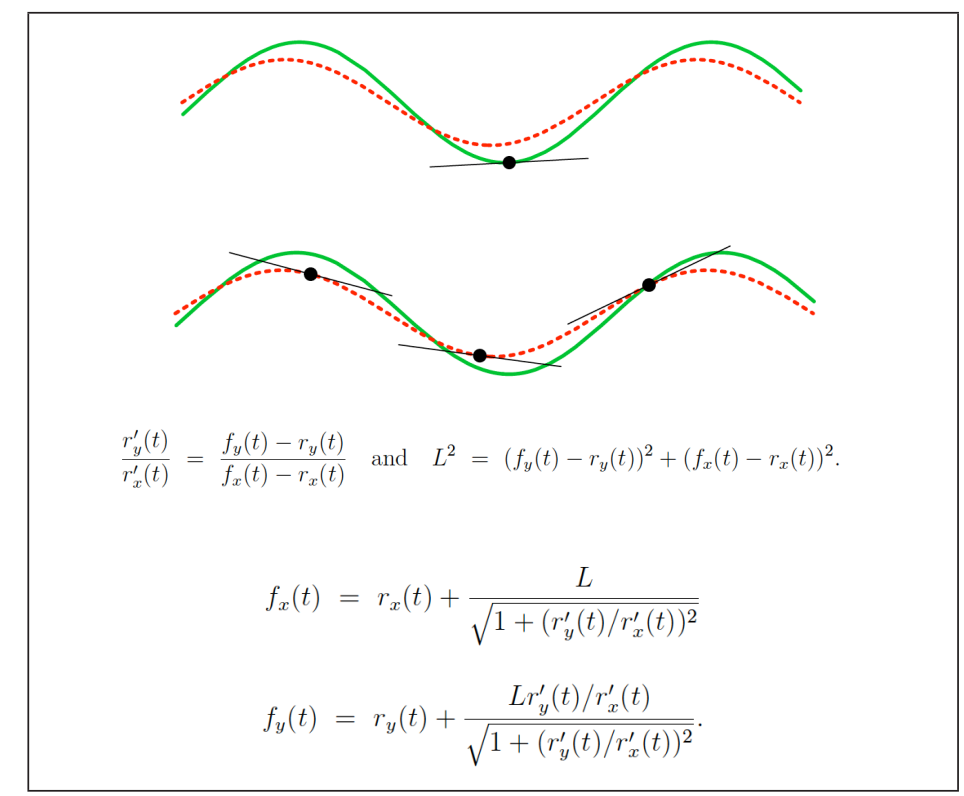

Figura 12. Propiedades geométricas de las curvas en las coordenadas de sus puntos (http://www.Math.UCSD.edu/ ebender/87/bicycle.pdf) 


\section{DISCUSIÓN}

Me gustaría concluir este artículo volviendo al concepto de génesis identitarias y de trayectorias, a las que se hace referencia en la introducción -y a lo largo de este artículo-. Las condiciones de trabajo de los docentes de matemáticas e investigadores en el campo se han modificado profundamente en los últimos años, debido principalmente a la digitalización de los medios de información y de comunicación. En esta etapa, veo tres preguntas fundamentales, que se vislumbran en este artículo, y mi experiencia de los últimos años en Francia, Brasil, China, México o Senegal, me ha demostrado que éstas son ampliamente compartidas.

- ¿Cómo combinar con provecho los recursos digitales y los recursos tangibles? (Por ejemplo, para apoyar las interacciones a partir de las simples hojas grandes de papel colgadas en la pared contra los sofisticados espacios virtuales en línea; para simular los trazos de la bicicleta sobre la arena, una memoria USB... o una bicicleta vs. una simulación en línea);

- ¿Cómo estimular la creatividad matemática en los grupos de estudiantes y de profesores? El ejemplo de la colaboración de Tom Apostol (teórico analítico de los números/analytic number theorist) y Mamikon Mnatsakanian (desarrollador del cálculo visual/visual calculus developper) es interesante, cómo el cruce productivo de dos trayectorias muy diferentes... En el proyecto europeo MC2 (creatividad matemática al cuadrado/ Mathematics creativity squared) se analizaron las profundas relaciones entre la creatividad social y la creatividad matemática, iqué habrá que estudiar detenidamente!

- ¿Cómo combinar la aportación de conocimientos nuevos a los estudiantes (y los profesores) con el apoyo a su propia actividad? Actualmente, estoy involucrado en un proyecto de investigación en Francia, PREMaTT (pensar en los recursos de los docentes en un tiempo de transición), que se basa en una red de escuelas, pensadas como auténticas "fábricas pequeñas". Son los mismos maestros los que proponen, intercambian, experimentan problemas y orquestaciones, y los investigadores juegan un papel de acompañamiento. Un proyecto prometedor...

Lo cual, desde mi punto de vista, vuelve particularmente crítico el desarrollo de marcos teóricos que sitúen en el centro de su reflexión las interacciones de los profesores con los recursos de su enseñanza, como el enfoque documental en la didáctica. Se celebró una conferencia internacional en Francia en 2018, para 
reflexionar sobre los programas de investigación que era necesario desarrollar (https://Resources-2018.sciencesconf.org/), y un libro reuniría estas propuestas (Trouche, Gueudet, \& Pepin, en vías de ser publicado).

Por último, me gustaría dar las gracias a la revista Educación Matemática y a Ana Isabel Sacristán por su invitación a publicar un artículo y hacerle llegar a la revista, con motivo de su $30^{\circ}$ aniversario, todos mis deseos de éxito al servicio de los docentes, de los estudiantes y del conocimiento.

\section{Agradecimientos}

Al Departamento de Matemática Educativa del CINVESTAV y a su director, Luis Moreno-Armella y a Armando Cuevas, por la invitación a una estancia de investigación de dos meses en 2017.

Al Freudenthal Institute y a Paul Drijvers, por su invitación a un seminario

A los profesores, investigadores y estudiantes que han nutrido este trabajo de investigación

A la revista que me ha invitado, a Ana Isabel Sacristán y Avenilde Romo, por la riqueza de sus interacciones en torno a este artículo.

\section{REFERENCIAS}

Adler, J. (2012). Knowledge resources in and for school mathematics teaching. In G. Gueudet, B. Pepin, \& L. Trouche (eds.), From Text to 'Lived' Resources: Mathematics Curriculum Materials and Teacher Development (pp. 3-22). NY: Springer.

Apostol, T., \& Mnatsakanian, M. (2012). New horizons in geometry. MAA.

Assis, C., Gitirana, V., \& Trouche, L. (2018). The Metamorphosis of resource systems of prospective teacher: from studying to teaching. In V. Gitirana, T. Miyakawa, M. Rafalska, S. Soury-Lavergne, \& L. Trouche (Eds.), Proceedings of the Re(s)sources International Conference (pp. 39-42). ENS de Lyon.

Bender, E.A. (2004). Sherlock Holmes and the bicycle tracks http://www.math.ucsd.edu/ ebender/87/bicycle.pdf

Conway, J., Doyle, P., Gilman, J., \& Thurston, B. (2006). Geometry and the imagination (https://math.dartmouth.edu/ doyle/docs/gi/gi.pdf).

Drijvers, P., \& Trouche, L. (2008). From artifacts to instruments: a theoretical framework behind the orchestra metaphor. In K. Heid \& G. Blume (Eds.), Research on Technology 
and the Teaching and Learning of Mathematics, Vol., Cases and perspectives (pp. 363-392), Charlotte, NC: Information Age.

Drijvers, P., Doorman, M., Boon, P., Reed, H., \& Gravemeijer, K. (2010). The teacher and the tool: instrumental orchestrations in the technology-rich mathematics classroom. Educational Studies in Mathematics, 75(2), 213-234.

Gueudet, G., \& Trouche, L. (2008). Du travail documentaire des enseignants : genèses, collectifs, communautés. Le cas des mathématiques. Education et didactique, 2(3), 7-33. Gueudet, G., \& Trouche, L. (2009a). Conceptions et usages de ressources pour et par les professeurs, développement associatif et développement professionnel, Les dossiers de l'ingénierie éducative, 65, 76-80.

Gueudet, G., \& Trouche, L. (2009b). Towards new documentation systems for mathematics teachers? Educational Studies in Mathematics, 71(3), 199-218.

Gueudet, G., Pepin, B., \& Trouche, L. (Eds.) (2012). From Text to 'Lived' Resources: Mathematics Curriculum Materials and Teacher Development. New York: Springer.

Gueudet, G., \& Trouche, L. (2012). Teachers' work with resources: documentation geneses and professional geneses. In G. Gueudet, B. Pepin, \& L. Trouche (eds.), From Text to 'Lived' Resources: Mathematics Curriculum Materials and Teacher Development (pp. 23-41). NY: Springer.

Gueudet, G., \& Trouche, L. (2016). Do Trabalho documental dos professores: gênesis, coletivos, comunidade. O caso da matemática. Revista de Educaçāo Matemática e Tecnológica Ibero-americana, 6(3), https://periodicos.ufpe.br/revistas/emteia/article/view/2243/1815

Guin, D., Joab, M., \& Trouche, L. (Eds.) (2008). Conception collaborative de ressources pour l'enseignement des mathématiques, l'expérience du SFoDEM (2000-2006), CDRom, INRP et IREM, Université Montpellier 2, disponible à http://www.math.univ-montp2.fr/sfodem/

Guin, D., \& Trouche, L. (1998). The Complex Process of Converting Tools into Mathematical Instruments. The Case of Calculators, The International Journal of Computers for Mathematical Learning, (3), 195-227.

Guin, D., \& Trouche, L. (dir.) (2002). Calculatrices symboliques. Faire d'un outil un instrument du travail mathématique : un problème didactique, Editions La Pensée sauvage, Grenoble.

Guin, D., \& Trouche, L. (2005). Distance training, a key mode to support teachers in the integration of ICT? In M. Bosch (Ed.), Proceedings of the Fourth European Conference on Research on Mathematics Education (pp. 1020-1029). FUNDEMI IQS - Universitat Ramon Llull, disponible à http://www.mathematik.uni-dortmund.de/ erme/CERME4/index.php

Hammoud, R. (2012). Le travail collectif des professeurs en chimie comme levier pour la mise en œuvre de démarches d'investigation et le développement des connaissances professionnelles. Contribution au développement de l'approche documentaire du didactique. $\mathrm{PhD}$, Université Lyon 1, accessible à https://tel.archives-ouvertes.fr/tel-00762964 
Hoyles, C., Kalas, I., Trouche, L., Hivon, L., Noss, R., \& Wilensky, U. (2010). Connectivity and Virtual Networks for Learning. In C. Hoyles, \& J.-B. Lagrange (Eds.), Mathematical Education and Digital Technologies: Rethinking the terrain (pp. 439-462). New York: Springer.

Monaghan, J., Trouche, L., \& Borwein, J. (2016). Tools and Mathematics: Instruments for Learning, New York, Springer.

MC2 (2017). Mathematical creativity squared, FP7 project http://www.mc2-project.eu/

Pastré, P. (2005). Genèse et identité. In P. Rabardel \& P. Pastré (dir.), Modèles du sujet pour la conception (pp. 231-260). Toulouse : Octarès.

Pédauque, R. T. (coll.) (2006). Le document à la lumière du numérique. Caen: C \& F éditions. Pepin, B., Choppin, J., Ruthven, K., \& Sinclair, N. (2017). Digital curriculum resources in mathematics education: foundations for change. ZDM- Mathematics Education, (5), 645-661.

Pepin, B., Xu, B., Trouche, L., \& Wang, C. (2016). Developing a deeper understanding of mathematics teaching expertise: Chinese mathematics teachers' resource systems as windows into their work and expertise. Educational studies in Mathematics, (3), 257-274, http://rdcu.be/koXk

PREMaTT (2018). http://ife.ens-lyon.fr/ife/recherche/groupes-de-travail/prematt

Rabardel, P. (1995). Les hommes et les technologies, approche cognitive des instruments contemporains. Paris : Armand Colin.

Rabardel, P., \& Bourmaud, G. (2005). Instruments et systèmes d'instruments. In P. Rabardel \& P. Pastré (dir.), Modèles du sujet pour la conception. Dialectiques activités développement (pp. 211-229). Toulouse: Octarès.

Remillard, J.T. (2005). Examining key concepts in research on teachers' use of mathematics curricula. Review of Educational Research, 75(2), 211-246.

Rocha, M. K., \& Trouche, L. (2016). Da produção coletiva de livros didáticos digitais aos usos feitos professores de Matemática: o caso do grupo francês Sésamath. Revista de Educaçāo Matemática e Tecnológica Ibero-americana, (3).

Salinas-Hernández, U., Sacristán, A. I., \& Trouche, L. (2018). Technology integration into mathematics classrooms: case study of a highschool teacher's use of GeoGebra. In V. Gitirana, T. Miyakawa, M. Rafalska, S. Soury-Lavergne, \& L. Trouche (Eds.), Proceedings of the Re(s)sources International Conference (pp. 88-91). ENS de Lyon.

Sanchez, M. (2010). Orquestación documentacional : herramienta para la estructuración y el análisis del trabajo documentacional colectivo en línea, Recherches en didactique des mathématiques, 30(3), 370-394.

Trouche, L. (2004). Managing the complexity of human/machine interactions in computerized learning environments: guiding students' command process through instrumental orchestrations. International Journal of Computers for Mathematical Learning, 9, 281-307. 
Trouche, L. (2005). An instrumental approach to mathematics learning in symbolic calculators environments. In D. Guin, K. Ruthven, \& L. Trouche (Eds.), The didactical challenge of symbolic calculators: turning a computational device into a mathematical instrument (pp. 137-162). N.Y.: Springer.

Trouche, L. (2009). Penser la gestion didactique des artefacts pour faire et faire faire des mathématiques : histoire d'un cheminement intellectuel, L'Educateur, 3, 35-38.

Trouche, L. (2018a). Understanding teachers' work throughout their interactions with resources. Invited lecture for the Freudenthal Institute, Utrecht University, April 19th.

Trouche, L. (2018b). Instrumentation in Mathematics Education. In S. Lerman (Ed.), Encyclopedia of Mathematics Education. N.Y.: Springer. doi:10.1007/978-3-319-77487-9_80-3.

Trouche, L. (2018c). Instrumentalization in Mathematics Education. In S. Lerman (Ed.), Encyclopedia of Mathematics Education. N.Y.: Springer. doi:10.1007/978-3-319-77487-9_100013-1.

Trouche, L., Drijvers, P., Gueudet, G., \& Sacristan, A. I. (2013). Technology-Driven Developments and Policy Implications for Mathematics Education. In A.J. Bishop, M.A. Clements, C. Keitel, J. Kilpatrick, \& F.K.S. Leung (Eds.), Third International Handbook of Mathematics Education (pp. 753-790). Springer.

Trouche, L., Gueudet, G., \& Pepin, B. (2018). Documentational approach to didactics. In S. Lerman (Ed.), Encyclopedia of Mathematics Education. N.Y.: Springer. doi:10.1007/978-3-319-77487-9_100011-1.

Trouche, L., Gueudet, G., \& Pepin, B. (Eds.) (to be published). Resources in Mathematics Teachers' Professional Activity. Springer series Advances in Mathematics Education.

Trouche, L., Trgalova, J., Loisy, C., \& Alturkmani, M. (2018). Ressources vivantes pour l'enseignement et l'apprentissage. Contribution des composantes IFÉ et S2HEP de I'ANR ReVEA. ENS de Lyon, https://hal.archives-ouvertes.fr/hal-01743212

Van den Heuvel-Panhuizen, M. (2003). The didactical use of models in realistic mathematics education : a example from a longitudinal trajectory on percentage, Educational Studies in Mathematics, 54, 9-35.

Vergnaud, G. (2009). The Theory of Conceptual Fields. Human Development, 52, 83-94.

Wang, C., Trouche, L., \& Pepin, B. (2018). An investigation of Chinese mathematics teachers' resources work and their professional development in collectives, In G. Schubring, L. Fan, \& V. Giraldo (Eds.). Proceeding of the second International Conference on Mathematics Textbooks, Research and Development. Rio, 8-11 May 2017.

LUC TROUCHE

Dirección: 15 parvis René-Descartes, BP 7000, 69342 Lyon cedex 07

Teléfono: $\quad$ +33 (0) 437376000 